\title{
Therapeutic Potential of CUDC-907 (Fimepinostat) for Hepatocarcinoma Treatment Revealed by Tumor Spheroids- based Drug Screening
}

Wei Liao

Zhujiang Hospital

Wanren Yang

Zhujiang Hospital

Yue Zhang

Zhujiang Hospital

Fanhong Zeng

Zhujiang Hospital

Jiecheng $\mathbf{X u}$

Zhujiang Hospital

Zhengming Yan

Zhujiang Hospital

Mingxin Pan

Zhujiang Hospital

Xiaoping $\mathrm{Xu}$

Zhujiang Hospital

Shuqin Zhou

Zhujiang Hospital

Min Zeng

Zhujiang Hospital

Jun Weng

Zhujiang Hospital

Xu Han

Zhujiang Hospital

Shao Li

Zhujiang Hospital

Yang Li

Zhujiang Hospital

Kangyan Liang

Zhujiang Hospital

Qing Peng

Zhujiang Hospital

Yi Gao ( $\nabla$ drgaoy@126.com )

Zhujiang Hospital, Southern Medical University https://orcid.org/0000-0003-3525-0133

\section{Research}

Keywords: Three-dimensional culture, drug screening, hepatocarcinoma, primary cells, CUDC-907 (fimepinostat) 
Posted Date: December 15th, 2020

DOI: https://doi.org/10.21203/rs.3.rs-125324/v1

License: @ (i) This work is licensed under a Creative Commons Attribution 4.0 International License. Read Full License 


\section{Abstract}

Background: Cancer is the second leading cause of death globally. However, most of the new anti-cancer agents screened by traditional drug screening methods fail in the clinic because of lack of efficacy. One of the reasons for this dilemma is that the two-dimensional (2D) culture cancer cell lines could not represent the in vivo cancer cells well. Fortunately, the development of a three-dimensional (3D) culture technique helps in this problem.

Methods: The high-throughput spheroid culture plate was fabricated by using 3D print technique and agarose. 4 hepatocarcinoma (HCC) cell lines were 3D cultured to screen 19 small molecular agents based on the spheroid culture plate. 3D cultured primary HCC cells and tumor-bearing mice model were established to verify the candidate anti-hepatocarcinoma agent. Cell function experiments and western blotting were conducted to explore the anti-hepatocarcinoma mechanism of the candidate agent.

Results: Based on the previous study, we established an in vitro 3D drug screening method by using our invented spheroid culture device and found that CUDC-907 can serve as a potent anti-hepatocarcinoma agent. The study data show that CUDC-907 (fimepinostat), a novel dual acting inhibitor of phosphoinositide 3-kinase (PI3K) and histone deacetylase (HDAC), has potent inhibitory effects on HCC cell lines and primary HCC cells in vitro, Animal studies have shown that CUDC-907 can also suppress HCC cells in vivo. Furthermore, we investigated the antitumor mechanism of CUDC-907 in HCC cells. We found that it inhibits the PI3K/AKT/mTOR pathway and downregulates the expression of c-Myc, leading to the suppression of HCC cells.

Conclusion: Our results suggest that CUDC-907 can be a candidate anti-HCC drug, and the 3D in vitro drug screening method based on our novel spheroid culture device is promising for drug screening.

\section{Background}

Globally, approximately 1 in 6 deaths are attributed to cancer, making it the second leading cause of death [1]. The great progress in molecular targeted therapy, in recent years, has successfully prolonged the survival time of cancer patients (especially in acute myeloid leukemia, breast, gastric, and colon cancers), showing a bright prospect [2]. However, many refractory cancers, such as hepatocellular carcinoma (HCC), still lack potent anti-tumor molecular agents. Therefore, substantial efforts are still needed to discover and screen new anti-cancer molecular agents for HCC treatment.

It should be mentioned that developing new drugs is a very expensive process. Relevant reports have pointed out that the preclinical and clinical development processes cost for one drug is about \$648 million to \$2.7 billion in USA [3, 4]. One of the main reasons for the high cost of drug development is the high risk of clinical failure of new drugs. Thus, choosing an effective preclinical model that can improve the predictive power in the drug screening process is an impactful measure to reduce the cost of drug development and greatly improve the successful treatment of cancer patients.

In vitro cancer models are used for drug screening, mainly including two-dimensional (2D) and three-dimensional (3D) cancer models. Traditionally, two-dimensional (2D) cultured immortal cancer cell lines are dominant in the research of antitumor agents because of their convenience in application. However, recent studies have shown that 2D cultured cells do not recapture the spatial architecture of in vivo tissues, changing the environmental stiffness and the distribution of nutrients, which affects the cell behavior and gene expression profile $[5,6]$. The extensive serial passages of cell lines generate genotypic and phenotypic alterations [7]. which may hinder the correct growth and response to external or internal stimuli. These results imply that the drug response of 2D-cultured cancer cell lines may not reflect the actual response of cancer cells in vivo.

Compared with the 2D cancer model, the 3D cancer model, which can mimic the in vivo cancer microenvironment and recapture the original cancer characteristics, shows great potential for in vitro drug screening [8]. Besides, primary cancer cells obtained from patients could maintain their primitive genetic background and features a long time [9]. Therefore, the 
combination of 3D culture and primary cancer cells may improve the accuracy of anti-tumor drug screening. In a previous study, we developed a novel spheroid culture system using 3D printing and agarose materials to promote the development and improve the accuracy of 3D in vitro dug screening. This 3D culture system could be used to form spheroid cultures for various cells, including primary HCC cells, and is inexpensive, standardized, and suitable for high-throughput drug screening [10].

HCC is one of the most lethal malignancies worldwide, but lacks potent molecular targeted anti-HCC agents [11, 12]. In this study, we used our invented spheroid culture system to screen 19 molecular inhibitors targeting the cell cycle and DNA damage (which are the main mechanism in tumor suppression), and identified a potential anti-HCC agent, CUDC-907. CUDC907, also known as Fimepinostat, is a synthetic, orally available, small molecule invented by Curis. It inhibits both phosphatidylinositol 3-kinase (PI3K) and histone deacetylase (HDAC) [13]. At present, CUDC-907 has not yet been approved by the FDA as a treatment option for any disease and is still undergoing clinical trials on some types of cancers. In preclinical studies, CUDC-907 has been proposed to have therapeutic potential in many hematopoietic malignancies and solid cancers, such as chronic lymphocytic leukemia, acute myeloid leukemia, thyroid cancer, and pancreatic adenocarcinoma [14-17]. However, it remains unclear whether CUDC-907 has an anti-HCC effect.

In this study, we found that the molecular inhibitor CUDC-907 can serve as a potential anti-HCC agent by using an in vitro 3D drug screening method on our invented spheroid culture system. We investigated the anti-tumor effect of CUDC-907 on HCC cell lines in vitro and in vivo. Primary HCC cells were used to verify the efficacy of CUDC-907 using a 3D method. Furthermore, we confirmed that CUDC-907 was able to inhibit the HDAC and PI3K/AKT/mTOR pathways and suppress the expression of C-MYC in HCC cells in vitro and in vivo, suggesting that this might be the main reason for its inhibitory effect on HCC. This study indicates that our invented spheroid culture system is a promising tool for drug screening and that CUDC-907 has the potential to be an anti-HCC agent in the future.

\section{Materials And Methods}

\subsection{Fabrication of agarose microwells in a 96-well plate}

The method for fabrication of agarose microwells in a 96-well plate was described in detail in a previous study [10]. Here is a simple illustration: (1) The stamp-like molds were designed and created using Solidwork software (Dassault Systems, Concord, USA). (2) The resin molds were printed using a Formlabs Phoenix Touch Pro UV-LED 3D printing system (Full Spectrum Laser, Las Vegas, USA). (3) After printing, pure alcohol was used to clean the resin residue on the molds. (4) Before use, the resin mold was sterilized with $75 \%$ alcohol or under an ultraviolet lamp for $1 \mathrm{~h}$. (5) $2 \%(\mathrm{w} / \mathrm{v}$, agarose/deionized water) melted $\left(80-100^{\circ} \mathrm{C}\right.$ ) agarose (111860, BioWest Regular, Hong Kong, China) was added to 96-well plates (Black Plate, Clear Bottom 96 Well Assay plate, 3603, Corning, NY, USA) (170 $\mu \mathrm{l} /$ well). (6) Before agarose solidification, the mold was used to shape the liquid agarose and pull out the mold when the agarose was cool. (7) Before using the agarose microwells, $170 \mu \mathrm{L}$ of Dulbecco's Modified Eagle's Medium (C11995500BT, DMEM, Gibco, Beijing, China) was added to saturate each agarose well. After $15 \mathrm{~min}$, the culture medium was removed, and the process was repeated 3 times. The agarose concave 96-well culture plate was then ready for use.

\subsection{HCC cell line culture and spheroid formation}

The human HCC cell lines HuH-7, BEL-7402, and SMMC-7721 were purchased from the Cell Bank of the Chinese Academy of Sciences, Shanghai, China. SNU-449 cells were purchased from ATCC (American Type Culture Collection). All cell lines were cultured in DMEM with 10\% fetal bovine serum (A3160801, Gibco). For spheroid formation, cells were seeded onto a homemade spheroid culture plate at a density of 1500 cells/ well. HCC cells were then cultured in DMEM with $10 \%$ fetal bovine serum and incubated at $37^{\circ} \mathrm{C}$ in a humidified atmosphere with $5 \% \mathrm{CO} 2$.

\subsection{Drug screening of HCC cell lines in spheroid culture}


The HCC cell lines were incubated for two days in 3D culture plates (1500 cell/well) and the drug sensitivity test was then performed (Fig. 1). In the first round of drug screening, 19 molecular inhibitors targeting the cell cycle and DNA damage were selected from an inhibitor screening library (HYCPK1971, MedchemExpress, New Jersey, USA). Specifically, inhibitors were diluted to $10 \mu \mathrm{M}$ with culture medium and then added into the spheroid culture well after 2-3 days of culture. Cell viability was measured using the CellTiter-Gloß 3D Cell Viability Assay (G9683, Promega Corporation, Madison, USA) according to the manufacturer's instructions, after 3 days of treatment, and then the inhibition ratio was calculated. Inhibitors with inhibition ratios above $85 \%$ were selected. After that, the second round of drug screening was executed. The inhibitors identified by the first-round screening were serially diluted to six concentrations with the culture medium, and the highest concentration was $10 \mu \mathrm{M}$. The drugs were added into the 3D culture wells and cells were treated for three days. Then, the cell activity of HCC spheroids and the inhibition ratio were measured and calculated. Finally, the inhibitor with the best inhibitory effect on HCC cells was chosen and the last round of drug screening was conducted. Four HCC cell lines were cultured in the homemade spheroid culture plate, and treated these cell spheroids with the last selected inhibitor serially diluted to 6 concentrations with culture medium for 3 days. Cell activity was measured and the inhibitory concentration of $50 \%$ (IC50) of this inhibitor on 4 different cells was calculated by using the Prohibit method with SPSS 20.0 software. Each assay was performed in triplicate.

\subsection{Isolation, culture, and drug sensitive test of human primary HCC and hepatic cells}

The HCC and liver samples were obtained with the consent of the patients and the hospital ethics committee (approval document number: 2017-GDEK-004). The isolation and culture method of primary HCC cells has been described previously [10]. For short, the isolation and culture processes are as follows: immediately after surgery, we first cut the HCC tumor into 3-4 slices and obtained specimens to avoid the necrotic area. Then, the specimens were immersed in DMEM and transported to the laboratory at $0{ }^{\circ} \mathrm{C}$. The specimens were rinsed 2-3 times with DMEM to remove the blood and were cut into small fragments ( $1 \mathrm{~mm} 3$ or smaller). The cut specimens were placed in Hank's Balanced Salt Solution (HBSS, 14025076, Gibco) containing $0.1 \%$ type IV collagenase (17104-019, Gibco) and digested for $40 \mathrm{~min}$ to $90 \mathrm{~min}$ at $37^{\circ} \mathrm{C}$. The resulting suspension was filtered through a 100- $\mu \mathrm{m}$ nylon filter and centrifuged at $50 \times \mathrm{g}$ for $3 \mathrm{~min}$ at $4{ }^{\circ} \mathrm{C}$. Then, the pellet was washed twice with HBSS. The final cell suspensions were cultured in T25 flasks (TCF001050, BIOFIL, Guangzhou, China) and hepatocyte culture medium (CC-3198, Lonza, Basel, Switzerland) at $37^{\circ} \mathrm{C}$ in a humidified incubator with $5 \% \mathrm{CO} 2$. The medium was changed at $24 \mathrm{~h}$ after seeding to remove dead cells and debris. For the isolation of normal hepatocyte, there were some details different from the isolation of HCC cells. Specifically, $0.1 \%$ type IV collagenase solution was warmed by thermostat water bath and keeps in $37^{\circ} \mathrm{C}$. Then the digest solution was slowly and continually injected in the different spots of liver tissue through disposable sterile injector for about 30 mines. Until the whole tissue was fully digested and become soft, the tissue was cut into pieces and rinsed in the digest solution. After that, all the digest solution was collected and filtered through a 100- $\mu \mathrm{m}$ nylon filter. The isolation and culture steps followed up were as same as the procedure in culturing primary HCC cells. After 2-3 days of culture, the primary cells were harvested and seeded onto homemade spheroid culture plates at a density of 1500 cells/well for drug sensitivity testing. The method of drug-sensitivity testing of CUDC-907 on primary cells is as mentioned above.

\subsection{Animal studies}

Male Balb/c nude mice (4-5-week old) were purchased from the Animal Experimental Center of Southern Medical University. The animal study was carried out in strict accordance with the Guidelines for the Care and Use of Laboratory Animals, China. All experimental protocols involving animals were approved by the Laboratory Animal Ethics Committee of Southern Medical University. The SMMC-7721 cells at a density of $5 \times 10^{6}$ in $0.2 \mathrm{~mL}$ of PBS were inoculated subcutaneously into the left flank of the nude mice. When the tumor volume reached approximately $100 \mathrm{~mm}^{3}$ at approximately 1 week, the mice were randomly assigned to the vehicle control $(n=5)$ and treated groups $(n=5)$, and they were treated with $30 \%$ propylene glycol or $300 \mathrm{mg} / \mathrm{kg}$ CUDC-907 by intragastric administration, three times in one week with 1- or 2-day intervals, respectively. The tumors and body weights of the mice were measured individually twice weekly. The volume of the tumor 
was calculated using the following formula: volume $=$ length $\times$ width $\times$ height $\times \pi / 6$. After treatment for 18 days, the mice were sacrificed after the final therapy. Tumors were removed and lysed for western blotting or fixed for immunohistochemistry.

\subsection{Calcein-AM/propidium iodide staining}

The cells were incubated in phosphate-buffered saline (PBS, C10010500BT, PBS, Gibco) with $2 \mu \mathrm{M}$ calcein-AM and $2.5 \mathrm{\mu g} / \mathrm{mL}$ PI (C326 and P346, Dojindo Chemical Technology, Shanghai, China) for $10 \mathrm{~min}$ at room temperature, and then the spheroids were examined and photographed under a fluorescence microscope.

\subsection{Flow cytometry - apoptosis}

SMMC-7721 and Huh-7 cells were treated with CUCC-907 of different concentrations for $36 \mathrm{~h}$. To detect apoptosis, the Annexin V FITC Apoptosis Detection Kit (AD10, DOJINDO, Shanghai, China) was used. Cells were stained with annexin V and propidium iodide (PI) for 30 min at room temperature in the dark, according to the manufacturer's instructions. Cells were kept on ice before analysis by flow cytometry (FACSVerse, BD Biosciences, California, USA).

\subsection{Flow cytometry - cell cycle}

SMMC-7721 and Huh-7 cells were treated with CUCC-907 of different concentrations for $24 \mathrm{~h}$. After the treatment, cells were washed with PBS, harvested, centrifuged 2 times at $1,200 \mathrm{rpm}$ at $4{ }^{\circ} \mathrm{C}$ for $5 \mathrm{~min}$, and resuspended in $300 \mu \mathrm{L}$ of sodium citrate buffer containing $0.1 \%$ Triton X-100, $100 \mu \mathrm{g} / \mathrm{ml}$ RNase, and $1 \mathrm{mg} / \mathrm{mL}$ DAPI. The cell cycle distribution was determined by flow cytometry analysis using a flow cytometer (FACSVerse, BD Biosciences). Each assay was repeated in triplicate, and the data were analyzed using ModfitLT (Verity Software House, Maine, USA).

\subsection{Western blotting}

Cells were pelleted by centrifugation, washed once with ice-cold PBS, and lysed on ice for 30 min using the cell lysis buffer (p0013, Beyotime, Shanghai, China) according to the manufacturer's extraction protocol. Protein quantitation was performed using the BCA Protein Assay Kit (ab102536, Abcam, Shanghai, China). A total of $30 \mu \mathrm{g}$ of protein was denatured in Laemli buffer at $95^{\circ} \mathrm{C}$ for $5 \mathrm{~min}$, and western blotting was performed using the Bio-Rad system (TGX 10-15\% gels). Transfer was performed using the Trans Blot turbo system (Bio-Rad) into PVDF membranes. Images were acquired using the Bio-Rad Imaging Chemidoc XPS + system. Secondary anti-rabbit and anti-mouse HRP-conjugated antibodies were purchased from Beyotime (A0208; A0216). Proteins were detected using the following antibodies: PARP1(66520-1-Ig, Proteintech, Wuhan, China); Caspase 3 (66470-2-lg, Proteintech); P21 (60214-1-Ig, Proteintech); Cyclin D1 (60186-1-lg, Proteintech); AKT (60203-2-Ig, Proteintech); AKT-phospho-S473 (66444-1-Ig, Proteintech); 4EBP1 (60246-1-Ig, Proteintech ); cMYC (10828-1-AP, Proteintech); Histone H3 (acetyl K9) (ab32129, Abcam); S6 Ribosomal Protein (AF6354, Affinity, Jiangsu, China); Phospho-S6 Ribosomal Protein (Ser235/236) (4858T, Shanghai, China); Phospho-4E-BP1 (S65) (YP0618, Immunoway, Texas, USA).

\subsection{Immunofluorescence analysis}

To identify the extracted primary cells, immunofluorescence analysis was performed. The primary cells were fixed with $4 \%$ paraformaldehyde for 10 min at room temperature, permeabilized with $0.1 \%$ Triton X-100 for 20 min at room temperature, and then incubated with $5 \%$ BSA and $0.5 \%$ Triton in TBS solution for $1 \mathrm{~h}$. The following primary antibodies were purchased from Abcam: anti-human serum albumin (ALB, ab207327), anti-human keratin 18 (CK-18, ab668), anti-human Arginase1 (ARG-1, ab212522), and anti-human alpha fetoprotein (AFP, ab169552). The samples were incubated with primary antibodies for $16 \mathrm{~h}$ at $4{ }^{\circ} \mathrm{C}$. The secondary antibodies were anti-mouse IgG conjugated with Alexa ${ }^{\circledR}$ Fluor 488 (Abcam, ab 150078) and goat anti-rabbit IgG conjugated with Alexa ${ }^{\circledR}$ Fluor 555 (Abcam, ab150117). The samples were incubated with secondary antibodies for $1 \mathrm{~h}$ and incubated with DAPI (Abcam) for $10 \mathrm{~min}$ at room temperature in the dark. All fluorescent images were obtained using a Leica fluorescence microscope. 


\subsection{Immunohistochemistry staining}

The xenograft tumor tissues were fixed with $4 \%$ formalin and cut into $4 \mu \mathrm{m}$ thin sections. For immunohistochemical staining, paraffin slides were deparaffinized and subjected to antigen retrieval using citrate sodium solution ( $\mathrm{pH}$ 6.0). The slides were then incubated in TBS solution containing $1 \%$ BSA and $0.5 \%$ Triton for $1 \mathrm{~h}$ at room temperature. Slides were incubated with primary antibodies Ki67 (ab15580, Abcam) and c-MYC (10828-1-AP, Proteintech) at 1:500 dilution overnight at $4{ }^{\circ} \mathrm{C}$. Endogenous peroxidase activity was blocked in $3 \%$ hydrogen peroxide/methanol buffer for $15 \mathrm{~min}$ at room temperature. Detection of bound antibodies was accomplished with the Streptavidin-Peroxidase kit (sp-9001, ZSGB-BIO, Beijing, China). Immunohistochemistry was independently assessed using the quick score method. Each specimen was assigned a score according to the intensity of the nucleic staining (no staining $=0$; weak staining $=1$; moderate staining $=2$; and strong staining $=3$ ), and the proportion of positive cells was estimated and given a percentage score on a scale from 1 to $6(1-4 \%=1 ; 5-19 \%=2 ; 20-39 \%=3 ; 40-59 \%=4 ; 60-79 \%=5 ;$ and $80-100 \%=6)$. The final immune-reactive score was determined by multiplying the intensity score by the extent of score of stained cells, ranging from a minimum of 0 to a maximum of 18.

\subsection{Statistical analysis}

All experiments were performed at least three times. The results are presented as mean \pm standard deviation. Statistical analysis was performed using two-tailed unpaired Student's t-tests. $P<0.05$ was considered statistically significant.

\section{Results}

\section{CUDC-907 has a potent inhibitory effect on proliferation of HCC cell lines in spheroid-based drug screening experiments}

In order to provide a cheaper, stable, and standardized tool for in vitro 3D drug screening, we invented a novel stamp-like resin mold that can shape the agarose to form a special 3D structure for spheroid culture. We designed a drug screening process based on this spheroid culture system (Fig. 1). HCC cells can form uniform spheroids in our homemade 3D culture system (Fig. 2 A). Using this 3D culture system, we screened 19 molecular inhibitors targeting the cell cycle and DNA damage by using the HuH-7 cell line, and screened out five potent agents (PF-670462, PIK-75, ETP-46464, Cl-994, and CUDC907) with inhibition ratios above $85 \%$ at $10 \mu \mathrm{M}$ (Fig. 2 B). We then tested the inhibitory ratios of the above five agents with different concentrations on the $\mathrm{HuH}-7$ cell line (Fig. $2 \mathrm{C}$ ) and calculated the IC50 of each agent. The results showed that the inhibitory potency of CUDC-907 was stronger than that of the other four agents. We then used 4 HCC cell lines (SMMC-7721, HuH-7, SNU-449, and BEL-7402) to examine the inhibitory efficacy of CUDC-907 through 3D method and compare its effect with sorafenib -the first-line anti-HCC drug. We found that the $\mathrm{IC}_{50}$ of CUDC-907 on HCC lines ranged from 0.4 to $35.1 \mathrm{nM}$, which was far lower than that of Sorafenib (whose IC I0 $_{50}$ values for these four HCC lines ranged from 3.0 to $9.8 \mathrm{uM}$ ) (Fig.2 D, Fig. $2 \mathrm{E}$ and Fig. $2 \mathrm{G}$ ). Afterward, we used calcein-AM/propidium iodide staining to visualize the inhibitory effect of CUDC907 and sorafenib. The data showed that CUDC-907 was able to kill $\mathrm{HuH}-7$ cells at a low concentration and its killing effect was much stronger than that of sorafenib (Fig. 2 F).

\section{CUDC-907 inhibits proliferation of primary HCC cells and the in vitro inhibitory efficacy is stronger than Sorafenib}

To provide convincing evidence for the inhibitory efficacy of CUDC-907 on HCC cells, we used primary cells to verify the effect of CUDC-907. In this experiment, we successfully extracted and cultured six cases of primary HCC cells from HCC patients (Table 1) and a case of primary hepatocyte from the adjacent normal liver tissue of a sareomatiod hepatocellular 
carcinoma patient. Five of the six cases of the HCC cells were stained with the specific antibody of hepatocarcinoma cells (CK-18, ALB, AFP, and ARG-1). The results showed that most of the cells extracted from cancer tissue were HCC cells (Fig. 3 A). Then, we cultured these primary cells in our homemade 3D culture plate, and the results showed that the cells formed uniform and regular spheroids after $48 \mathrm{~h}$ (Fig. 3 B). After primary cells formed regular spheroids, 3D cultured primary cells from 7 cases were treated with CUDC-907 for 3 days, and primary HCC spheroids of 4 in 6 cases were treated with sorafenib (the first-line anti-HCC drug) for 3 days for comparison. The inhibition curves showed that CUDC-907 has a high inhibitory efficacy on primary HCC cells, and its efficacy is stronger than that of sorafenib; however, the inhibitory effect of CUDC-907 on primary hepatocyte is much lower even in the highest drug concentration (Fig. $3 \mathrm{C}$ ). The $\mathrm{IC}_{50}$ of CUDC-907 on six cases of primary HCC cells were different, ranging for $1.7 \mathrm{nM}$ to $130.03 \mathrm{nM}$, which were far lower than $\mathrm{IC}_{50}$ of Sorafenib (> 5000 nM, Fig.3 D). In addition, the calcein-AM/propidium iodide staining experiment revealed that CUDC-907 with a series of concentrations from 3.91-500 nM was able to kill primary HCC cells from HCC-3 patients, but the killing ability of CUDC-907 on primary hepatocyte is not obvious (Fig.3 E).

Table 1

Donor characteristics at the time of HCC resection

\begin{tabular}{|c|c|c|c|c|c|c|}
\hline $\begin{array}{l}\text { Patient } \\
\text { characteristics }\end{array}$ & HCC-1 & $\mathrm{HCC}-2$ & HCC-3 & HCC-4 & HCC-5 & HCC-6 \\
\hline Age/sex & $27 /$ female & $69 /$ male & $52 /$ male & $51 /$ male & $69 /$ male & $64 /$ male \\
\hline HBsAg & Positive & Positive & Positive & Positive & Positive & Positive \\
\hline $\begin{array}{l}\text { Serum HBV } \\
\text { DNA }\end{array}$ & $\begin{array}{l}7.08 \times 10^{2} \\
\mathrm{IU} / \mathrm{mL}\end{array}$ & $\begin{array}{l}3.05 \times 10^{5} \\
\mathrm{IU} / \mathrm{mL}\end{array}$ & - & $\begin{array}{l}4.36 \times 10^{5} \\
\mathrm{IU} / \mathrm{mL}\end{array}$ & - & $\begin{array}{l}1.08 \times 10^{4} \\
\mathrm{IU} / \mathrm{mL}\end{array}$ \\
\hline $\begin{array}{l}\text { Anti-HCV } \\
\text { antibody }\end{array}$ & Negative & Negative & Negative & Negative & Negative & Negative \\
\hline $\begin{array}{l}\text { Serum a- } \\
\text { fetoprotein }\end{array}$ & $26.8 \mu \mathrm{g} / \mathrm{L}$ & $4 \mu \mathrm{g} / \mathrm{L}$ & $2.8 \mu \mathrm{g} / \mathrm{L}$ & $2590 \mu \mathrm{g} / \mathrm{L}$ & $2.9 \mu \mathrm{g} / \mathrm{L}$ & $144.2 \mu \mathrm{g} / \mathrm{L}$ \\
\hline $\begin{array}{l}\text { HCC } \\
\text { pathologic } \\
\text { diagnosis }\end{array}$ & $\begin{array}{l}\text { Poorly } \\
\text { differentiated } \\
\text { hepatocellular } \\
\text { carcinoma }\end{array}$ & $\begin{array}{l}\text { Highly } \\
\text { differentiated } \\
\text { hepatocellular } \\
\text { carcinoma }\end{array}$ & $\begin{array}{l}\text { Moderately } \\
\text { differentiated } \\
\text { hepatocellular } \\
\text { carcinoma }\end{array}$ & $\begin{array}{l}\text { Highly } \\
\text { differentiated } \\
\text { hepatocellular } \\
\text { carcinoma }\end{array}$ & $\begin{array}{l}\text { Moderately } \\
\text { differentiated } \\
\text { hepatocellular } \\
\text { carcinoma }\end{array}$ & $\begin{array}{l}\text { Moderately } \\
\text { differentiated } \\
\text { hepatocellular } \\
\text { carcinoma }\end{array}$ \\
\hline
\end{tabular}

\section{CUDC-907 inhibits the growth of HCC xenografts in vivo}

We next established a xenograft HCC model to evaluate the antitumor effect of CUDC-907 in vivo. The xenograft HCC model was established by subcutaneously injecting the human HCC cell line SMMC-7721 into nude mice. Until the tumor volume reached approximately $100 \mathrm{~mm}^{3}$, SMMC-7721 xenograft-bearing mice were treated with $300 \mathrm{mg} / \mathrm{kg}$ CUDC-907 three times a week. After the final treatment on day 18 , all the nude mice were sacrificed, and tumors were resected, imaged, measured, and weighed as shown in Fig. 4. Figure 4A shows that there were only four tumors resected from the drug-treated mice because the tumor from one of the treated mice grew too large and ruptured. Therefore, the data from this mouse were excluded. The results of animal experiments showed that CUDC-907 treatment significantly reduced tumor growth in the xenograft HCC model compared to the vehicle group (Fig. 4 C, D), and the body weight of mice showed no significant reduction (Fig. 4 B). Taken together, these findings suggest that CUDC-907 has a potential therapeutic effect on HCC xenografts. 


\section{CUDC-907 inhibits proliferation, causes G2/M arrest, and induces apoptosis in HCC cells}

Flow cytometry was used to evaluate changes in the cell cycle and apoptosis of HCC cells. SMMC-7721 and HuH-7 cells were treated with 10, 100, and $500 \mathrm{nM}$ CUDC-907 for $24 \mathrm{~h}$ for cell cycle assessment and $36 \mathrm{~h}$ for apoptosis assessment. CUDC-907 arrested SMMC-7721 and HuH-7 cells at the G2/M phase and strongly induced cell apoptosis in a concentrationdependent manner (Fig. 5 A and Fig. 5 B). Moreover, the colony assay validated the inhibitory effect of CUDC-907 on HCC cell lines (Fig. 5 C). Furthermore, key factors involved in cell apoptosis and cell cycle arrest were analyzed by western blotting. CUDC-907 markedly upregulated p21 (CDKN1A) and cleaved poly (ADP-ribose) polymerase, which was accompanied by decreases in the corresponding precursors, and CUDC-907 downregulated Caspase-3 and cyclin D1 (Fig. 5 D). These results suggested that CUDC-907 may inhibit HCC cell proliferation in part through cell cycle arrest and/or induction of cell apoptosis.

\section{CUDC-907 inhibits HDAC and PI3K/AKT/mTOR pathway, and suppress c-Myc function in HCC cells}

CUDC-907 is a dual PI3K and HDAC inhibitor, and previous studies have demonstrated that HDAC inhibitors act synergistically with PI3K inhibitors to inhibit tumor growth in an MYC-dependent manner in different types of cancer [14, 1820] (Fig.6 A). According to previous research on CUDC-907, the downregulation of c-Myc protein is an early event induced by CUDC-907 treatment, resulting in the growth restriction of c-Myc-driven cancers [21]. Importantly, HCC is a type of refractory cancer with predominant c-Myc overexpression [22], and the proliferation of HCC can be inhibited with a low concentration of CUDC-907, as suggested by the aforementioned results. To explore the mechanisms underlying this inhibition, we evaluated the expression of H3K9ac, the targets of PI3K and c-Myc in HCC cell lines and HCC xenograft tissue. The HCC cell lines SMMC-7721 and HuH-7 were treated with CUDC-907 at various concentrations for $24 \mathrm{~h}$. Western blotting analysis revealed that CUDC-907 upregulated H3K9ac levels and reduced the expression of p-AKT, p-S6, and p4EBP1(the targets of $\mathrm{PI} 3 \mathrm{~K} / \mathrm{AKT} / \mathrm{mTOR}$ pathway), and suppresses the expression of c-Myc in HCC cell lines (Fig. 5 B). In addition, western blotting analysis in HCC xenograft tissue also confirmed the downregulation of c-Myc upon treatment with CUDC-907. Immunohistochemistry analysis of the xenograft HCC tumor showed that the cell proliferation biomarker Ki67 levels and cMyc level in CUDC-907-treated group were downregulated compared to those in the untreated group (Fig. 6 D).

\section{Discussion}

Globally, cancer is currently the second leading cause of death and is expected to be responsible for approximately 9.6 million deaths in 2018 [23]. Owing to the unprecedented understanding of the molecular pathways that drive the development and progression of human cancers, novel targeted therapies have become an exciting new development for anti-cancer medicine. The researches on targeted therapies achieved a great progress that the application of targeted agents tremendously improve the survival time of partial patients with acute myeloid leukemia, breast, gastric, and colon cancers. However, when it comes to some refractory cancers, such as HCC, there is still a hug gap. Hepatocellular carcinoma is the sixth most common malignancy and the fourth leading cause of cancer-related deaths worldwide [24]. In recent years, the first line targeted drugs for HCC are sorafenib and its alternative lenvatinib. However, the anticancer efficacy of sorafenib is unsatisfactory because it only prolongs the overall survival (OS) period by approximately three months compared with placebo. The efficacy of lenvatinib is better than that of sorafenib, but the improvement is limited [25]. Thus, there is an urgent need to identify new potent anticancer drugs for HCC patients.

When it comes to drug screening for drug discovery and development, enormous amounts of money and time must be spent to obtain clinically approved drugs [26]. Even after the long and costly process to identify potential compounds, 80\% of drugs failed during clinical trials and the most common reason for failure was lack of efficacy [27]. Thus, choosing a more 
effective cancer model that has higher predictive power for anticancer drug screening can greatly improve the success rate of drug discovery and reduce the cost of the screening process. In recent years, the rapid development of 3D culture techniques has provided various pre-clinical models for drug screening. Compared with the traditional drug screening model of flattening (2D) cultured cancer cell lines, 3D cultured cancer models have unique advantages in mimicking the microenvironment and 3D structure of in vitro tumors, and are good at maintaining the functional characteristics of original cells $[27,28]$. Therefore, the 3D cultured cancer model has higher predictive power than the 2D model and is better for drug screening.

Spheroid culture is a type of 3D culture technology that has been developed for nearly three decades [29]. This technique is characterized by its low technical difficulty and cost, and it can well imitate the three-dimensional structure and metabolic function of tumors in vivo [30,31]. Thus, in a previous study, we developed a novel spheroid culture device for anti-tumor drug screening [10]. We used the 3D printing technique to print a resin mold with a fine structure on a micrometer-scale, and then we used the printed mold to shape the agarose substrate in the cell culture well of an ordinary 96-well plate to obtain a special structure, such as a micro-well and liquid exchanging platform. Finally, makes the ameliorated 96-well plate realizing the function of high-throughput 3D culture of cancer cells. Our invented 3D culture device is low cost, reusable, suitable for various cells including primary cancer cells, and is effective in controlling the size and shape of cultured spheroids, meaning that it is a proper tool in 3D drug screening. Therefore, in this study, we designed a set of schemes to screen the anti-HCC drugs by using our homemade 3D culture devise for the aims that find out a potential anti-HCC molecular targeted agent and hope to establish an effective method for in vitro 3D drug screening.

First, we conducted a three-round 3D drug screening on HCC cell lines using our homemade spheroid culture device, and finally screened out CUDC-907 as a potential anti-HCC agent from 19 inhibitors. The results of the drug sensitivity test showed that CUDC-907 has a potent inhibitory effect on HCC cell lines at a low concentration and its efficacy is obviously better than that of the first-line anti-HCC drug sorafenib. Furthermore, functional experiments showed that CUDC-907 inhibited proliferation, caused G2/M arrest, and induced apoptosis in HCC cells. The above evidence suggests that CUDC907 is a promising anti-HCC agent. CUDC-907, a dual PI3K/HDAC inhibitor, has been proposed to have therapeutic potential in many hematopoietic malignancies and solid cancers, such as chronic lymphocytic leukemia, acute myeloid leukemia, thyroid cancer, and pancreatic adenocarcinoma [14-17]. It has been recently granted Fast Track designation for the treatment of adults with relapsed or refractory diffuse large B-cell lymphoma by the US Food and Drug Administration [17]. Importantly, it is the first time that our research team found that CUDC-907 has a potent inhibitory effect on HCC cells.

Cancer cell lines are the oldest and most widely used models in cancer research because of their low cost and convenience in manipulation. Due to their ability to grow infinitely, they are well applicable in high-throughput screenings. However, the preparation of cell lines from a tumor results in loss of the 3D in vivo structure and diversity of cell populations; thus, these models only partly represent the origin tumor [32]. Besides, the long-term in vitro culture causes genetic drift and is easily cross-contaminated [33]. These disadvantages significantly limit the predictive power of cancer cell line models in drug screening. Fortunately, the improvement of the culture technique of primary cancer cells helps in these problems. Primary cancer cells maintain the original genetic background and different subgroups of cancer cells; therefore, primary tumor cells are more representative and suitable for drug screening [34-36]. Based on these findings, in this study, we extracted primary HCC cells for the 3D drug-sensitive test. Seven cases of primary cells including six cases of HCC and one case of hepatocyte were successfully extracted and cultured. The results of the drug sensitivity test showed that the inhibitory efficacy of CUDC907 on different patient-derived primary cells varied. The $\mathrm{IC}_{50}$ of CUDC-907 in different cases of primary HCC ranged from 1.7 to $103 \mathrm{nM}(0.86 \sim 52.38 \mathrm{ng} / \mathrm{mL})$, which is much lower than the $\mathrm{IC}_{50}$ of sorafenib, and the killing ability of CUDC-907 in primary hepatocyte is weak. These results suggest that the in vitro inhibitory effect of CUDC-907 on primary HCC cells is stronger than sorafenib but has individual difference in drug responses.

Although a previous report pointed out that the peak plasma concentration of CUDC-907 was around $5 \mathrm{ng} / \mathrm{mL}$, the concentration of CUDC-907 in tumor samples could reach approximately $69.5 \mathrm{ng} / \mathrm{g}$ [37]. Whether CUDC-907 is effective for

Page $10 / 26$ 
HCC treatment in vivo remains unknown. We next conducted an animal study to further prove the effect of CUDC-907 in vivo. Initially, we planned to establish patient-derived xenograft (PDX) mouse models by subcutaneous engraftment of tumor species. However, this experiment failed as the engrafted tissues did not grow in vivo. For substitution, we established an SMMC-7721 HCC xenograft. The results of animal experiments suggested that CUDC-907 could significantly suppress the growth of HCC tumors in vivo. All of these evidences supports the opinion that CUDC-907 might be a potential anti-HCC agent and the outcome of the experiment implies that the 3D in vitro drug screening method we used in this study is a valuable method for drug screening and discovery.

Previous reports have shown that CUDC-907 is a dual-acting inhibitor of PI3K and HDAC, and exhibits predominant anticancer effects in c-Myc-driven tumors [38, 39]. The c-Myc oncoprotein is an essential transcription factor that regulates the expression of many genes involved in cell growth, proliferation, and metabolic pathways [40]. The deregulation and enhancement of c-Myc play an essential role in the carcinogenesis and progression of virous cancer, including HCC [41]. In order to have a profound understanding of the anti-HCC effect of CUDC-907, we investigated the expression level of possible target molecules of CUDC-907. We used western blotting to investigate the impact of CUDC-907 on HDAC, PI3K, and c-Myc in HCC cells. Western blotting of HCC cell lines and xenograft tissues showed that CUDC-907 can inhibit the HDAC and $\mathrm{PI3K} / \mathrm{AKT} / \mathrm{mTOR}$ pathway and suppress the expression of c-MYC. It is worth mentioning that the PI3K/AKT/mTOR pathway is also a central regulator of various oncogenic processes including cell growth, proliferation, metabolism, and angiogenesis in HCC [42]. Thus, the dual inhibition of c-Myc and PI3K/AKT/mTOR may be the main reason that contributes to the potent effect of CUDC-907 on HCC.

\section{Conclusions}

In summary, the 3D spheroid-based drug screening we established in this experiment presents a valuable application in new anti-tumor drug screening and personalized treatment. The data in this study suggested that CUDC-907 might be a promising drug for HCC treatment. PDX studies and clinical research are still needed to validate its efficacy in HCC treatment.

\section{Abbreviations}

2D: two-dimensional

3D: three-dimensional

HCC: hepatocarcinoma

PI3K: phosphoinositide 3-kinase

HDAC: histone deacetylase

IC50: inhibitory concentration of $50 \%$

PBS: phosphate-buffered saline

ALB: albumin

CK-18: keratin 18

ARG-1: Arginase1

AFP: alpha fetoprotein

OS: overall survival 
PDX: patient-derived xenograft

\section{Declarations}

\section{Ethics approval and consent to participate}

The animal study was carried out in strict accordance with the Guidelines for the Care and Use of Laboratory Animals, China. All experimental protocols involving animals were approved by the Laboratory Animal Ethics Committee of Southern Medical University. The HCC and liver samples were obtained with the consent of the patients and the hospital ethics committee (approval document number: 2017-GDEK-004).

\section{Consent for publication}

The corresponding author and all the co-authors have agreed to the publication of the manuscript to Journal of Experimental and Clinical Cancer Research as a research article and declare that they have no conflict of interest as to the results presented.

\section{Availability of data and materials}

The datasets used and analyzed during the current study are available from the corresponding author on reasonable request.

\section{Competing interests}

The authors declare no conflict of interest.

\section{Funding}

This work was supported by the National Key R\&D Program of China (2018YFA0108200, 2018YFC1106400); the National Natural Science Foundation of China (31972926); The Natural Science Foundation of Guangdong Province (2014A030312013, 2018A030313128, 2018A030313214); Guangdong key research and development plan (2019B020234003); Science and Technology Program of Guangzhou (201803010086).

\section{Contributions}

WL and WY carried out molecular/cell biology experiments and wrote the manuscript. YZ, FZ, JX and ZY conducted the animal experiment. MP, XX and SZ provided the clinical samples. MZ, JW and XH performed statistical analyses. SL, YL and $\mathrm{KL}$ revised the article. YG and QP supervised the study and revised the manuscript. The author(s) read and approved the final manuscript.

\section{Acknowledgements}

The authors thank the staff of the Heart Center Laboratory, Zhujiang Hospital, for providing some of the experimental equipment. 


\section{References}

1. Bray F, Ferlay J, Soerjomataram I, et al. Global cancer statistics 2018: GLOBOCAN estimates of incidence and mortality worldwide for 36 cancers in 185 countries. CA Cancer J Clin. 2018;68:394-424.

2. Lee YT, Tan YJ, Oon CE. Molecular targeted therapy: Treating cancer with specificity. Eur J Pharmacol. 2018;834:188196.

3. Prasad V, Mailankody S. Research and Development Spending to Bring a Single Cancer Drug to Market and Revenues After Approval. JAMA Intern Med. 2017;177:1569-1575.

4. DiMasi JA, Grabowski HG, Hansen RW. Innovation in the pharmaceutical industry: New estimates of R\&D costs. J Health Econ. 2016;47:20-33.

5. Skardal A, Mack D, Atala A, et al. Substrate elasticity controls cell proliferation, surface marker expression and motile phenotype in amniotic fluid-derived stem cells. J Mech Behav Biomed Mater. 2013;17:307-316.

6. Brancato V, Oliveira JM, Correlo VM, et al. Could 3D models of cancer enhance drug screening? Biomaterials. 2019;232:119744.

7. Tsuji K, Kawauchi S, Saito S, et al. Breast cancer cell lines carry cell line-specific genomic alterations that are distinct from aberrations in breast cancer tissues: comparison of the $\mathrm{CGH}$ profiles between cancer cell lines and primary cancer tissues. BMC Cancer. 2010;10:15.

8. Ryu NE, Lee SH, Park H. Spheroid Culture System Methods and Applications for Mesenchymal Stem Cells. Cells. 2019;8.

9. Song Y, Kim JS, Kim SH, et al. Patient-derived multicellular tumor spheroids towards optimized treatment for patients with hepatocellular carcinoma. J Exp Clin Cancer Res. 2018;37:109.

10. Liao W, Wang J, Xu J, et al. High-throughput three-dimensional spheroid tumor model using a novel stamp-like tool. J Tissue Eng. 2019;10:1543348704.

11. Llovet JM, Montal R, Sia D, et al. Molecular therapies and precision medicine for hepatocellular carcinoma. Nat Rev Clin Oncol. 2018;15:599-616.

12. Li W, Deng R, Liu S, et al. Hepatitis B virus-related hepatocellular carcinoma in the era of antiviral therapy: The emerging role of non-viral risk factors. Liver Int 2020.

13. Qian C, Lai CJ, Bao R, et al. Cancer network disruption by a single molecule inhibitor targeting both histone deacetylase activity and phosphatidylinositol 3-kinase signaling. Clin Cancer Res. 2012;18:4104-4113.

14. Fu XH, Zhang X, Yang H, et al. CUDC-907 displays potent antitumor activity against human pancreatic adenocarcinoma in vitro and in vivo through inhibition of HDAC6 to downregulate c-Myc expression. Acta Pharmacol Sin. 2019;40:677688.

15. Kotian S, Zhang L, Boufraqech M, et al. Dual Inhibition of HDAC and Tyrosine Kinase Signaling Pathways with CUDC907 Inhibits Thyroid Cancer Growth and Metastases. Clin Cancer Res. 2017;23:5044-5054.

16. Chen Y, Peubez C, Smith V, et al. CUDC-907 blocks multiple pro-survival signals and abrogates microenvironment protection in CLL. J Cell Mol Med. 2019;23:340-348.

17. Li X, Su Y, Madlambayan G, et al. Antileukemic activity and mechanism of action of the novel PI3K and histone deacetylase dual inhibitor CUDC-907 in acute myeloid leukemia. Haematologica. 2019;104:2225-2240.

18. Simmons JK, Michalowski AM, Gamache BJ, et al. Cooperative Targets of Combined mTOR/HDAC Inhibition Promote MYC Degradation. Mol Cancer Ther. 2017;16:2008-2021.

19. Mondello P, Derenzini E, Asgari Z, et al. Dual inhibition of histone deacetylases and phosphoinositide 3-kinase enhances therapeutic activity against B cell lymphoma. Oncotarget. 2017;8:14017-14028. 
20. Pei Y, Liu KW, Wang J, et al. HDAC and PI3K Antagonists Cooperate to Inhibit Growth of MYC-Driven Medulloblastoma. Cancer Cell. 2016;29:311-323.

21. Sun K, Atoyan R, Borek MA, et al. Dual HDAC and PI3K Inhibitor CUDC-907 Downregulates MYC and Suppresses Growth of MYC-dependent Cancers. Mol Cancer Ther. 2017;16:285-299.

22. Xu Z, Xu M, Liu P, et al. The mTORC2-Akt1 Cascade Is Crucial for c-Myc to Promote Hepatocarcinogenesis in Mice and Humans. Hepatology. 2019;70:1600-1613.

23. Seebacher NA, Stacy AE, Porter GM, et al. Clinical development of targeted and immune based anti-cancer therapies. J Exp Clin Cancer Res. 2019;38:156.

24. Llovet JM, Zucman-Rossi J, Pikarsky E, et al. Hepatocellular carcinoma. Nat Rev Dis Primers. 2016;2:16018.

25. Liu Z, Lin Y, Zhang J, et al. Molecular targeted and immune checkpoint therapy for advanced hepatocellular carcinoma. J Exp Clin Cancer Res. 2019;38:447.

26. Moffat JG, Rudolph J, Bailey D. Phenotypic screening in cancer drug discovery - past, present and future. Nat Rev Drug Discov. 2014;13:588-602.

27. Kondo J, Inoue M. Application of Cancer Organoid Model for Drug Screening and Personalized Therapy. Cells. $2019 ; 8$.

28. Cheng Y, Wang Y, Kang YZ, et al. In vitro culture of tumour-derived hepatocytes in decellularised whole-liver biological scaffolds. Digestion. 2013;87:189-195.

29. Landry J, Bernier D, Ouellet C, et al. Spheroidal aggregate culture of rat liver cells: histotypic reorganization, biomatrix deposition, and maintenance of functional activities. J Cell Biol. 1985;101:914-923.

30. Dilworth C, Hamilton GA, George E, et al. The use of liver spheroids as an in vitro model for studying induction of the stress response as a marker of chemical toxicity. Toxicol In Vitro. 2000;14:169-176.

31. Li AP, Colburn SM, Beck DJ. A simplified method for the culturing of primary adult rat and human hepatocytes as multicellular spheroids. In Vitro Cell Dev Biol. 1992;28A:673-677.

32. Unger FT, Witte I, David KA. Prediction of individual response to anticancer therapy: historical and future perspectives. Cell Mol Life Sci. 2015;72:729-757.

33. Meijer TG, Naipal KA, Jager A, et al. Ex vivo tumor culture systems for functional drug testing and therapy response prediction. Future Sci OA. 2017;3:0190.

34. Broutier L, Mastrogiovanni G, Verstegen MM, et al. Human primary liver cancer-derived organoid cultures for disease modeling and drug screening. Nat Med. 2017;23:1424-1435.

35. Allinen M, Beroukhim R, Cai L, Brennan C, et al. Molecular characterization of the tumor microenvironment in breast cancer. Cancer Cell. 2004;6:17-32.

36. Sharma S, Neale MH, Di Nicolantonio F, et al. Outcome of ATP-based tumor chemosensitivity assay directed chemotherapy in heavily pre-treated recurrent ovarian carcinoma. BMC Cancer. 2003;3:19.

37. Younes A, Berdeja JG, Patel MR, et al. Safety, tolerability, and preliminary activity of CUDC-907, a first-in-class, oral, dual inhibitor of HDAC and $\mathrm{PI} 3 \mathrm{~K}$, in patients with relapsed or refractory lymphoma or multiple myeloma: an open-label, doseescalation, phase 1 trial. Lancet Oncol. 2016;17:622-631.

38. Sun K, Atoyan R, Borek MA, et al. Dual HDAC and PI3K Inhibitor CUDC-907 Downregulates MYC and Suppresses Growth of MYC-dependent Cancers. Mol Cancer Ther. 2017;16:285-299.

39. Ferreira AC, De-Freitas-Junior JC, Morgado-Díaz JA, et al. Dual inhibition of histone deacetylases and phosphoinositide 3-kinases: effects on Burkitt lymphoma cell growth and migration. J Leukoc Biol. 2016;99:569-578.

40. Farrell AS, Sears RC. MYC degradation. Cold Spring Harb Perspect Med 2014;4.

41. Srivastava J, Siddiq A, Gredler R, et al. Astrocyte elevated gene-1 and c-Myc cooperate to promote hepatocarcinogenesis in mice. Hepatology. 2015;61:915-929. 
42. Ashworth RE, Wu J. Mammalian target of rapamycin inhibition in hepatocellular carcinoma. World J Hepatol 2014;6:776-782.

\section{Figures}

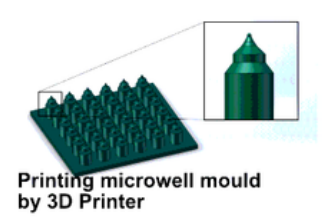

by 3D Printer

First round of drug screening

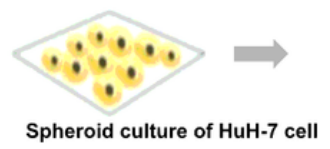

Second round of drug screening
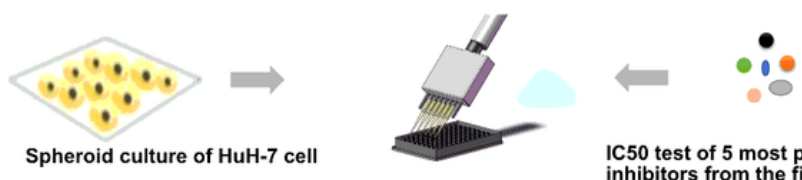

IC50 test of 5 most potent inhibitors from the first round screening

Third round of drug screening

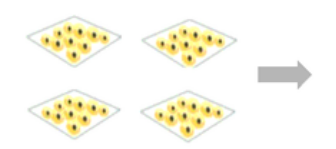

Spheroid culture of $4 \mathrm{HCC}$ cell lines
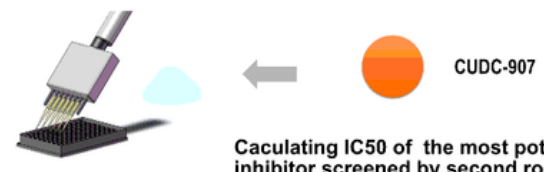

Caculating IC50 of the most potent inhibitor screened by second roud screening in different HCC cells

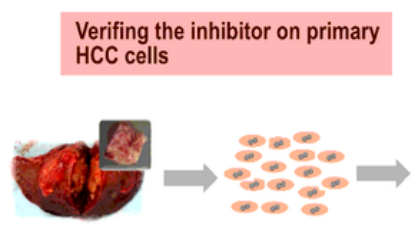

Extracting and spheroid culturing primary HCC cells to conduct the drug
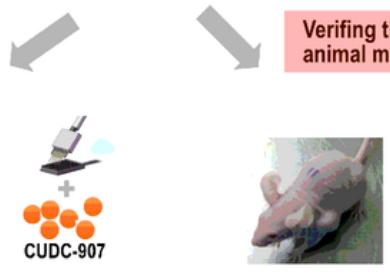

Verifing the inhibitor on HCC animal modle

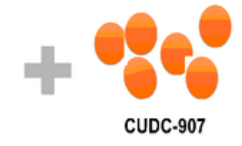

\section{Figure 1}

The schematic diagram of drug screening using spheroid-based in vitro models and in vivo experiment 


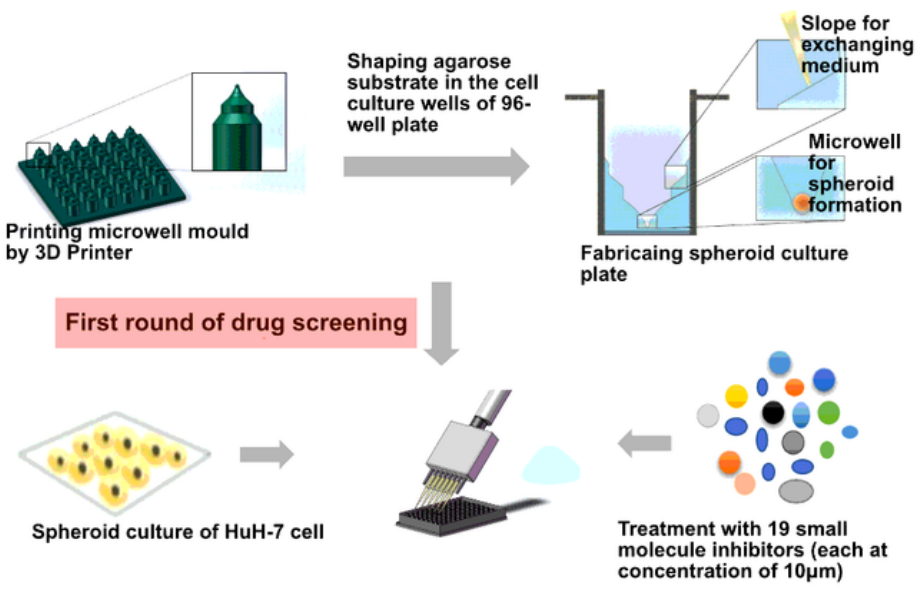

Second round of drug screening

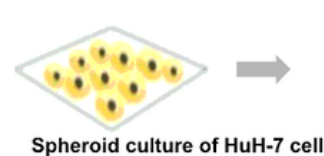

Spheroid culture of HuH-7 cell

Third round of drug screening

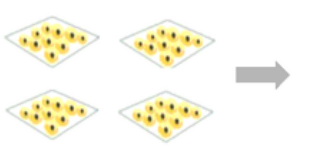

Spheroid culture of $4 \mathrm{HCC}$ cell lines
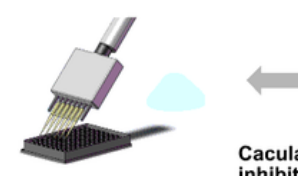

Caculating IC50 of the most potent inhibitor screened by second roud screening in different HCC cells

Verifing the inhibitor on HCC animal modle
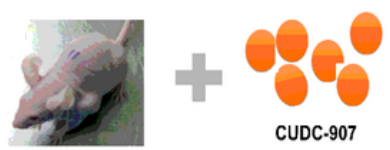

Extracting and spheroid culturing primary HCC cells to conduct the drug
sensitive test on screened inhibitor

\section{Figure 1}

The schematic diagram of drug screening using spheroid-based in vitro models and in vivo experiment 


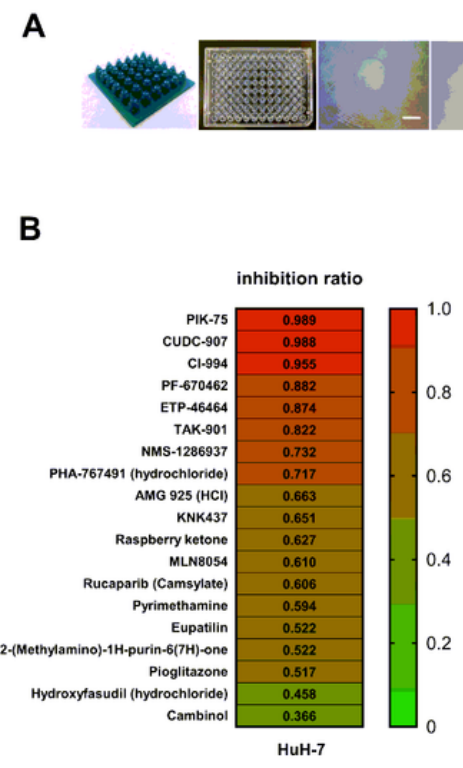

$\mathbf{F}$

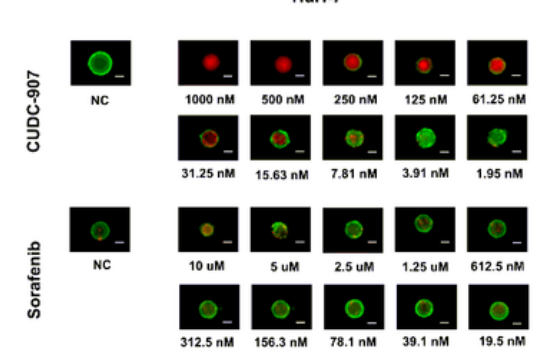

C
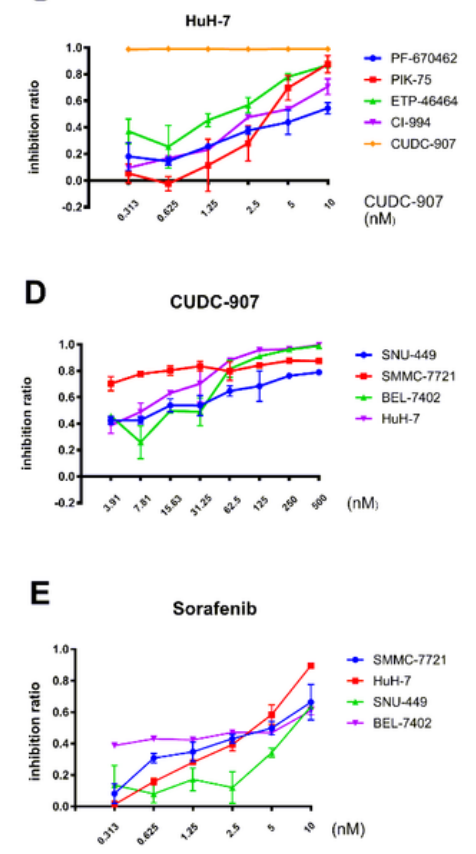

G

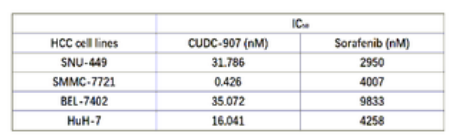

\section{Figure 2}

CUDC-907 was screened as a potential anti-HCC agent by in vitro 3D drug screening. A The entity of 3D printed resin mold and the ameliorated 96-well plate with shaped agarose substrate. There is a micro-well in the agarose substrate and HCC cell lines can form uniform spheroid in the micro-well. B The first round of drug screening. HuH-7 cells were cultured in the homemade 3D culture plate and were treated of 19 inhibitors in the concentration of $10 \mathrm{uM}$ for 3 days. The inhibition rate was measured by ATP assay. C-E Inhibitory curve of different inhibitors on HCC cell lines. HCC cell lines were cultured in homemade spheroid culture devise and treated with different agents for 3 days. The inhibition rate was measured by ATP assay. F Calcein-AM/propidium iodide staining of $\mathrm{HuH}-7$ spheroids. The $\mathrm{HuH}-7$ cells were treated with different concentration of CUDC-907 and sorafenib for 3 days. G The IC50 of sorafenib and CUCU-907 on 4 HCC cell lines. Scale bar = $200 \mathrm{uM}$. 


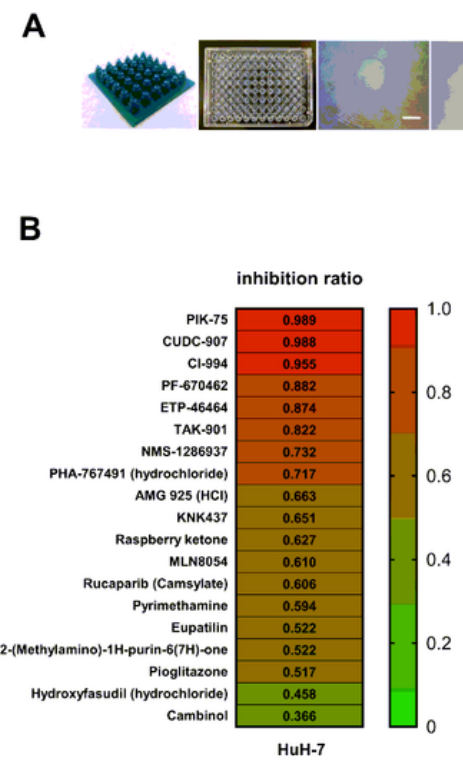

$\mathbf{F}$

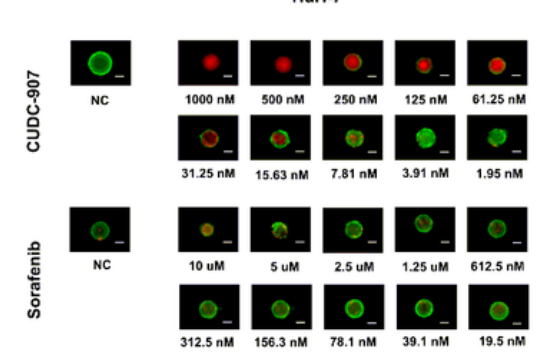

C
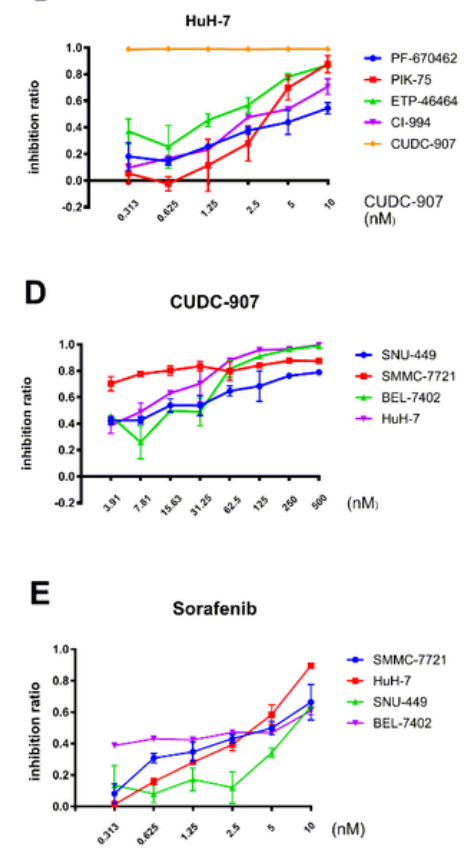

G

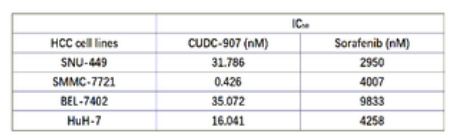

\section{Figure 2}

CUDC-907 was screened as a potential anti-HCC agent by in vitro 3D drug screening. A The entity of 3D printed resin mold and the ameliorated 96-well plate with shaped agarose substrate. There is a micro-well in the agarose substrate and HCC cell lines can form uniform spheroid in the micro-well. B The first round of drug screening. HuH-7 cells were cultured in the homemade 3D culture plate and were treated of 19 inhibitors in the concentration of $10 \mathrm{uM}$ for 3 days. The inhibition rate was measured by ATP assay. C-E Inhibitory curve of different inhibitors on HCC cell lines. HCC cell lines were cultured in homemade spheroid culture devise and treated with different agents for 3 days. The inhibition rate was measured by ATP assay. F Calcein-AM/propidium iodide staining of $\mathrm{HuH}-7$ spheroids. The $\mathrm{HuH}-7$ cells were treated with different concentration of CUDC-907 and sorafenib for 3 days. G The IC50 of sorafenib and CUCU-907 on 4 HCC cell lines. Scale bar = $200 \mathrm{uM}$. 
A

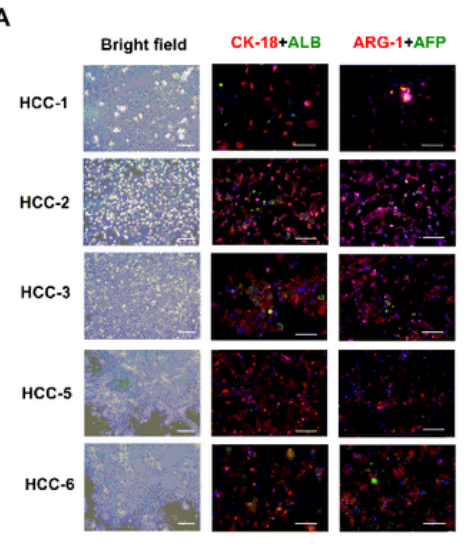

B

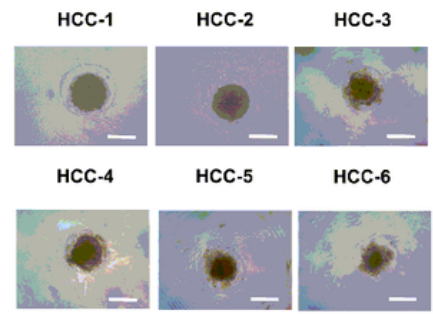

Hepatocyte

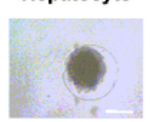

C
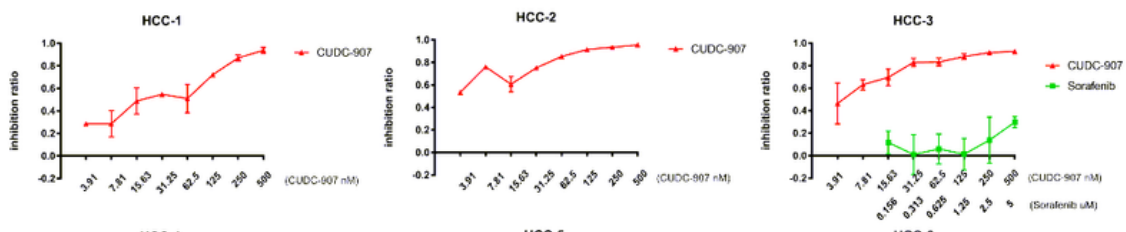

HCC-4
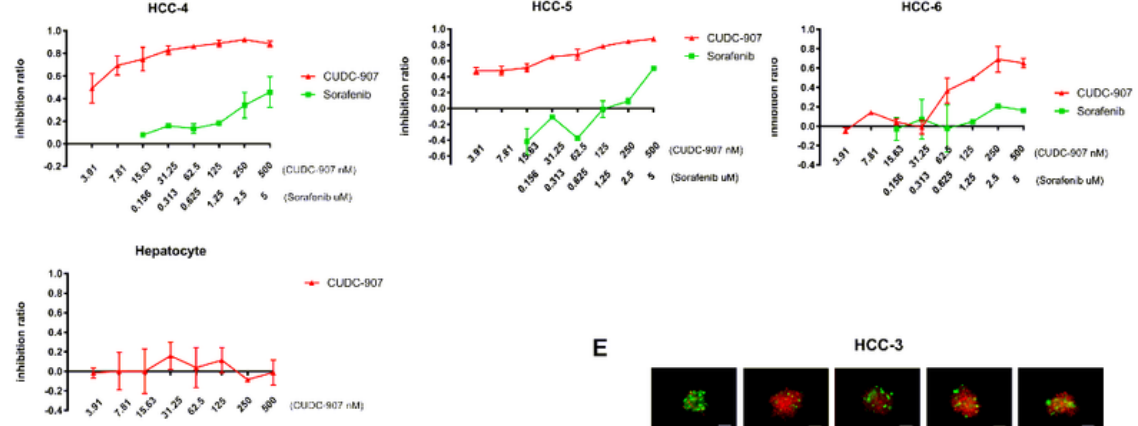

E

D
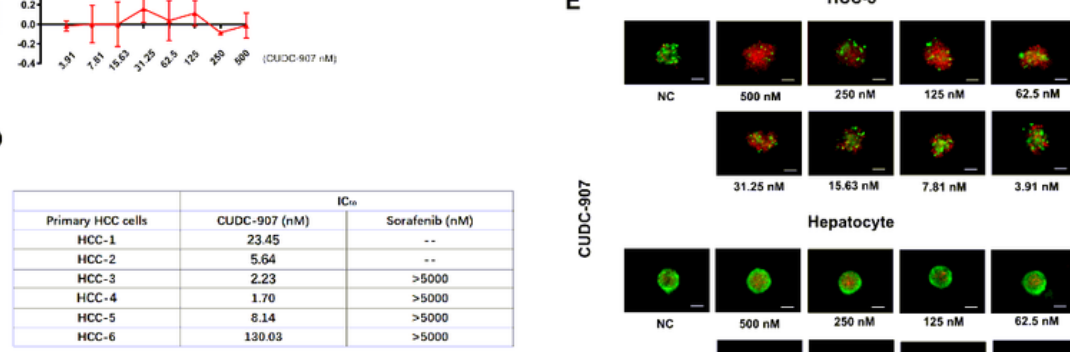

$500 \mathrm{nM}$

_

${ }_{2}{ }_{2}$

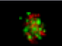

宊

Hepatocyte

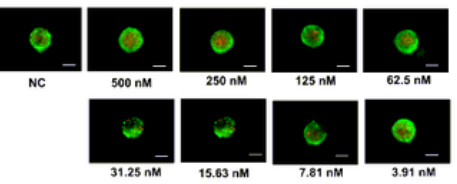

\section{Figure 3}

The drug sensitivity test of primary cell spheroid. A AFP, albumin, ARG-1 and CK-18 immunostaining of primary cells to examine the cellular origin of primary HCC cells. B Primary cells cultured in homemade 3D culture devise for 3 days. C Inhibitory curve of CUDC-907 and sorafenib on primary cell spheroid. Primary cell spheroids were treated with CUDC-907 and sorafenib for 3 days. D The IC50 of CUDC-907 and Sorafenib on primary HCC spheroids. E Calcein-AM/propidium iodide staining of primary cell spheroids. The primary cells were treated with CUDC-907 for 3 days. Scale bar $=200 \mathrm{uM}$. 
A

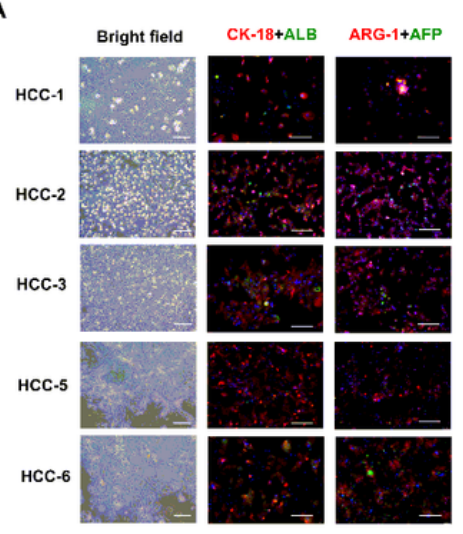

B

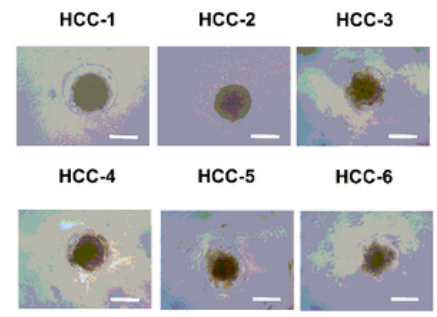

Hepatocyte

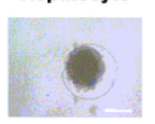

C
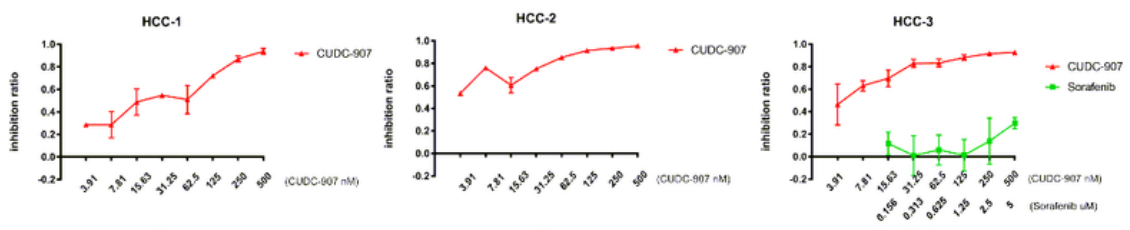

HCC-4
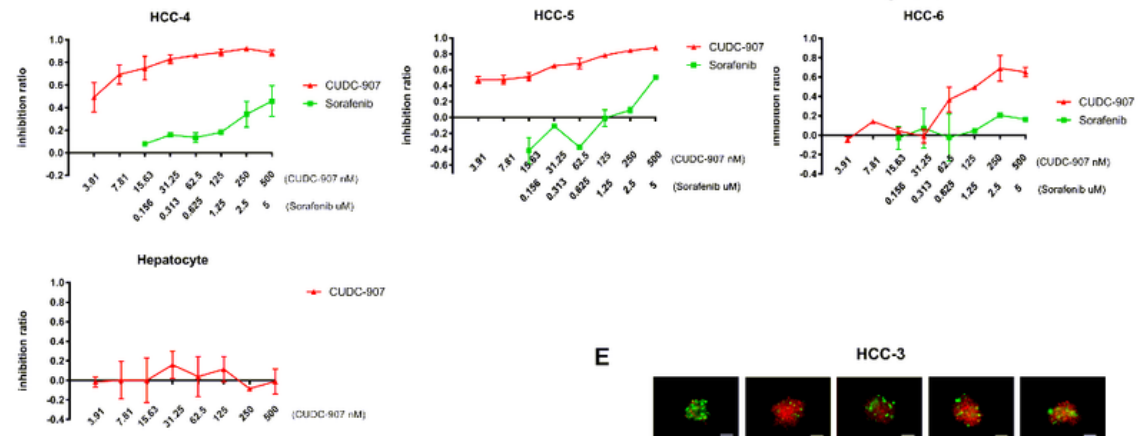

E

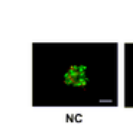

D

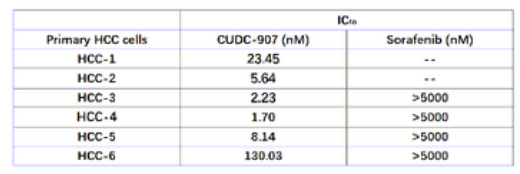

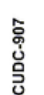

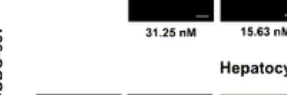

HCC-3
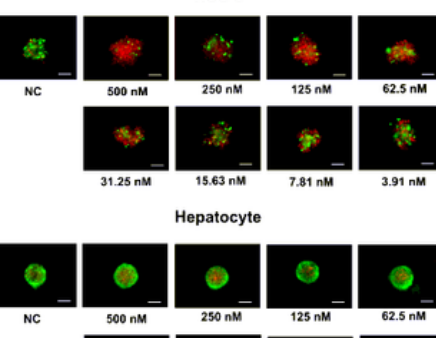

$500 \mathrm{nM}$

$250 \mathrm{nM}$

5

这

Hepatocyte
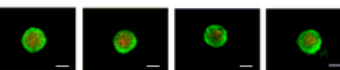

$500 \mathrm{~nm}$

\begin{tabular}{cc}
$250 \mathrm{~nm}$ & $125 \mathrm{~nm}$ \\
\hline & \\
\hline $15.63 \mathrm{nM}$ & \\
\hline
\end{tabular}

$\theta$

\section{Figure 3}

The drug sensitivity test of primary cell spheroid. A AFP, albumin, ARG-1 and CK-18 immunostaining of primary cells to examine the cellular origin of primary HCC cells. B Primary cells cultured in homemade 3D culture devise for 3 days. $C$ Inhibitory curve of CUDC-907 and sorafenib on primary cell spheroid. Primary cell spheroids were treated with CUDC-907 and sorafenib for 3 days. D The IC50 of CUDC-907 and Sorafenib on primary HCC spheroids. E Calcein-AM/propidium iodide staining of primary cell spheroids. The primary cells were treated with CUDC-907 for 3 days. Scale bar $=200 \mathrm{uM}$. 
A

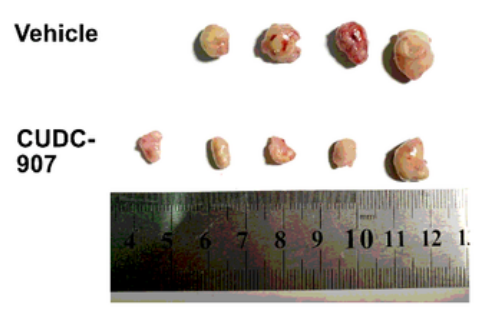

B

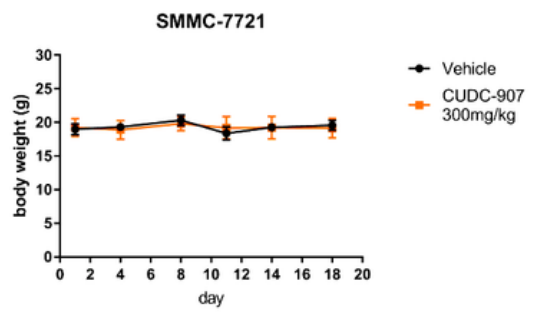

D

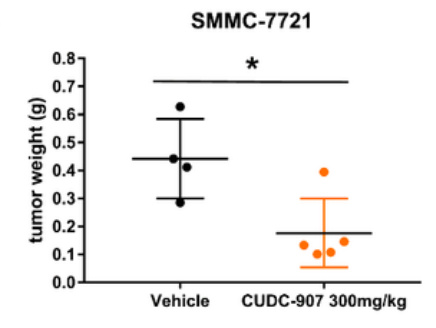

\section{Figure 4}

CUDC-907 inhibits HCC cancer growth in vivo. A Tumor morphology. After 18 days, all nude mice were sacrificed, and the tumors were resected to be imaged. $B$ and $C$ The curve of body weight and tumor volume in 18 days of treatment with CUDC-907 or vehicle. D Tumor weigh after 18 days of treatment. ${ }^{\star} \mathrm{p}<0.05,{ }^{*} \mathrm{p}<0.001$. 
A

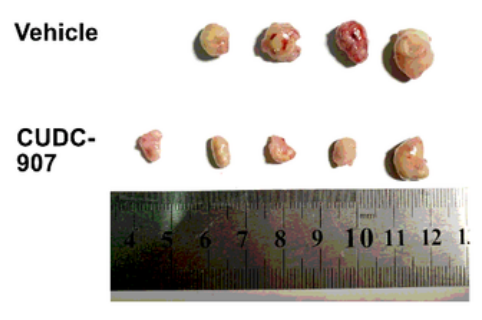

B

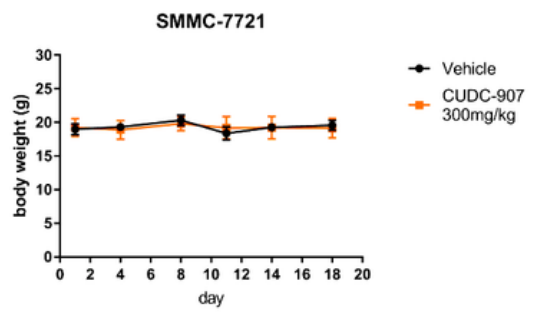

D

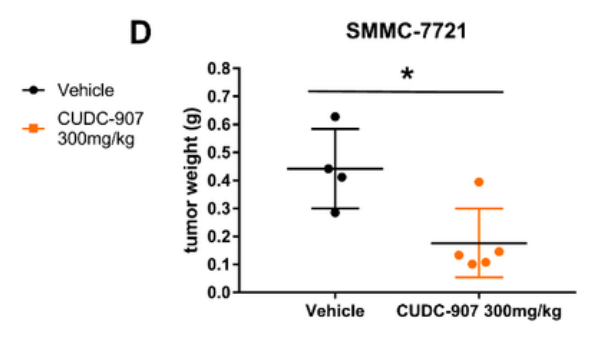

C

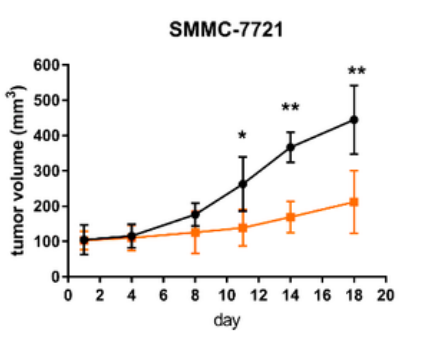

\section{Figure 4}

CUDC-907 inhibits HCC cancer growth in vivo. A Tumor morphology. After 18 days, all nude mice were sacrificed, and the tumors were resected to be imaged. $B$ and $C$ The curve of body weight and tumor volume in 18 days of treatment with CUDC-907 or vehicle. D Tumor weigh after 18 days of treatment. ${ }^{\star} p<0.05,{ }^{\star} * \mathrm{p}<0.001$. 
A
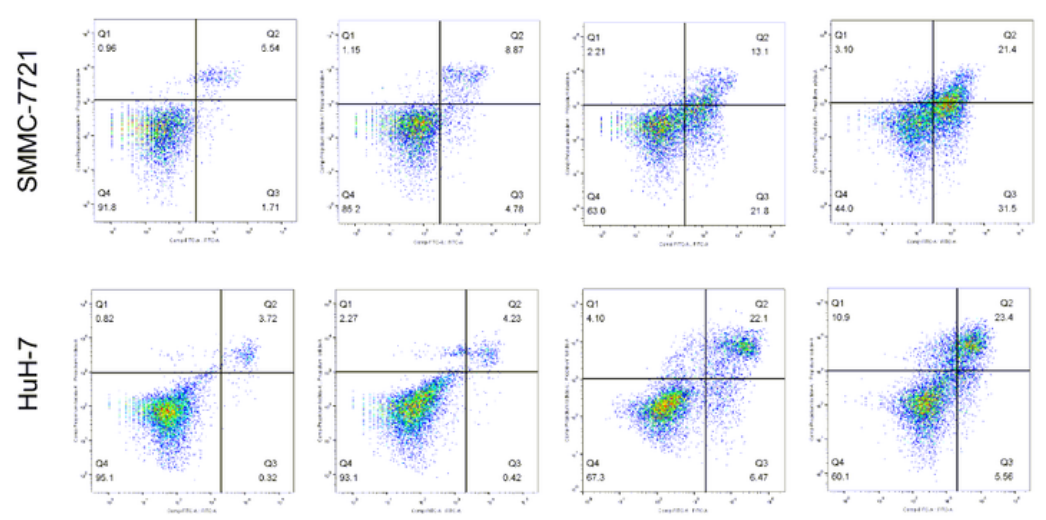

$\begin{array}{llll}\text { CUDC-907 NC } & 10 \mathrm{nM} & 100 \mathrm{nM} & 500 \mathrm{nM}\end{array}$

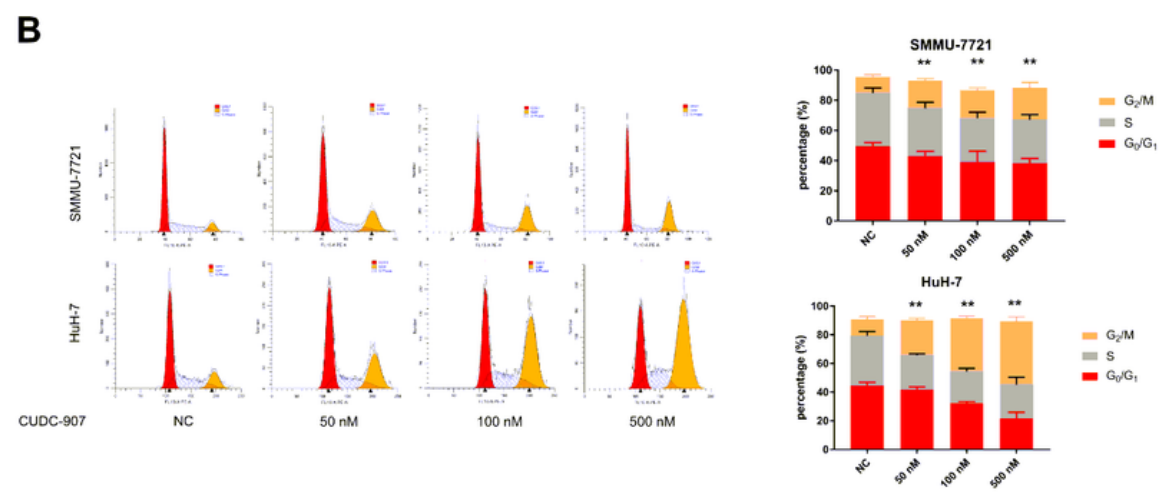

C

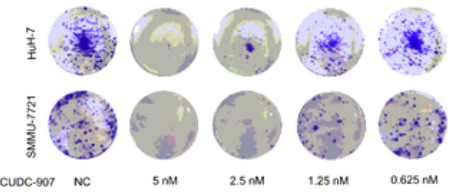

\section{D}
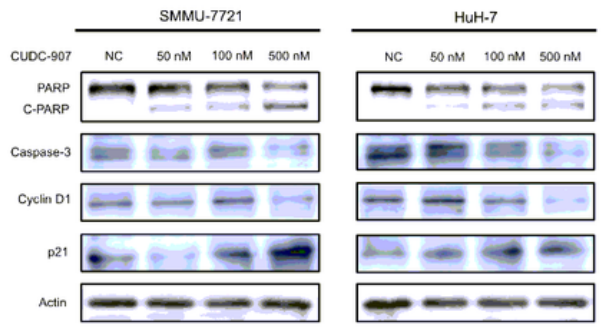

\section{Figure 5}

CUDC-907 inhibits proliferation, causes G2/M arrest, and induces apoptosis in HCC cells. A Cell apoptosis detected by Flow

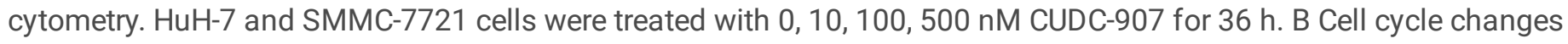
detected by Flow cytometry. HuH-7 and SMMC-7721 cells were treated with 0, 10, 100, 500 nM CUDC-907 for 24h. C Colonyformation assays. HuH-7 and SMMC-7721 cells were measured with crystal violet staining after treatment with CUDC-907 at 5 to $20 \mathrm{nM}$ for 14 days. D HuH-7 and SMMC-7721 cells were collected for Western blotting with the indicated antibodies after treatment with CUDC-907 for 48 h. ${ }^{*} p<0.05$, ** $p<0.001$. 
A
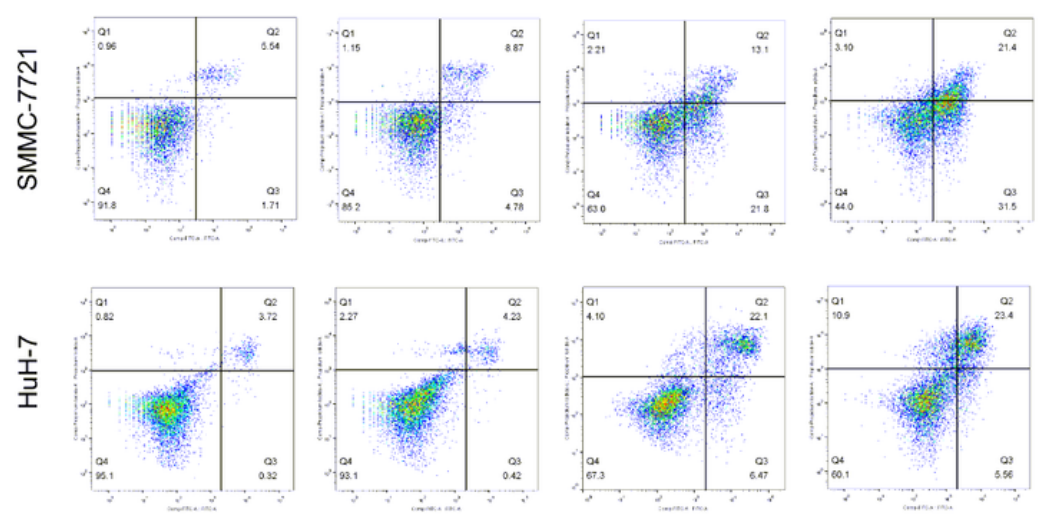

$\begin{array}{llll}\text { CUDC-907 NC } & 10 \mathrm{nM} & 100 \mathrm{nM} & 500 \mathrm{nM}\end{array}$

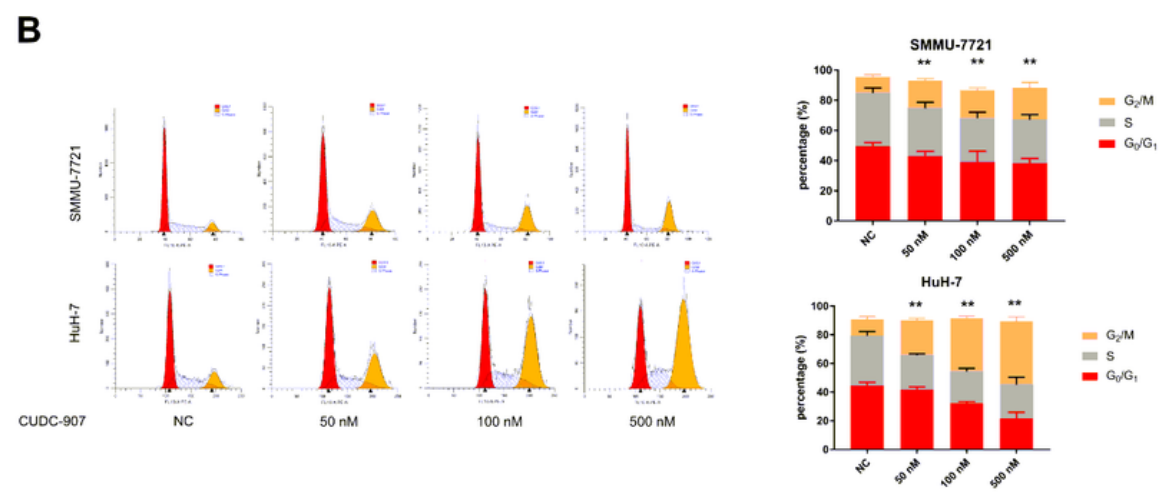

C

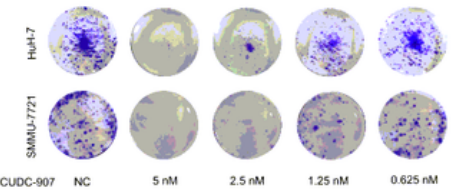

\section{D}
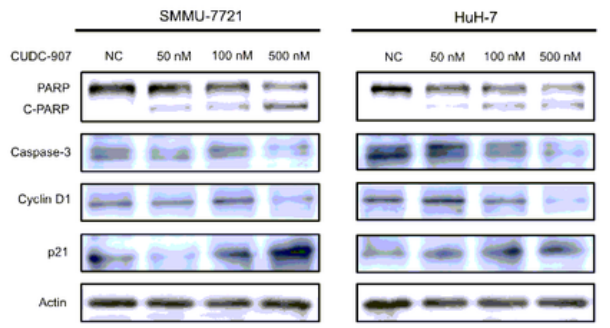

\section{Figure 5}

CUDC-907 inhibits proliferation, causes G2/M arrest, and induces apoptosis in HCC cells. A Cell apoptosis detected by Flow

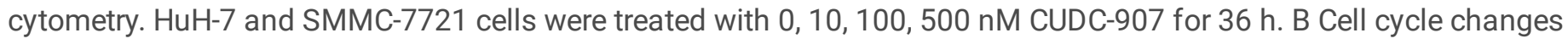
detected by Flow cytometry. HuH-7 and SMMC-7721 cells were treated with 0, 10, 100, 500 nM CUDC-907 for 24h. C Colonyformation assays. HuH-7 and SMMC-7721 cells were measured with crystal violet staining after treatment with CUDC-907 at 5 to $20 \mathrm{nM}$ for 14 days. D HuH-7 and SMMC-7721 cells were collected for Western blotting with the indicated antibodies after treatment with CUDC-907 for 48 h. ${ }^{*} p<0.05$, ** $p<0.001$. 
A

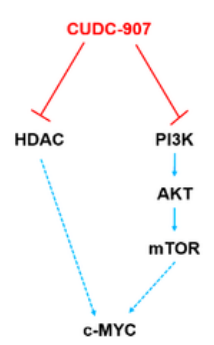

B

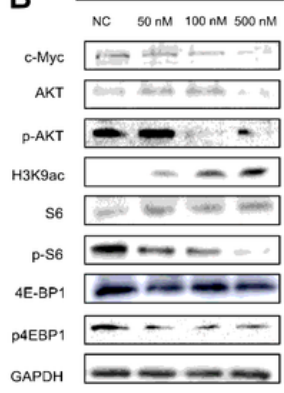

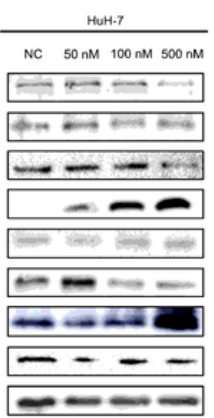
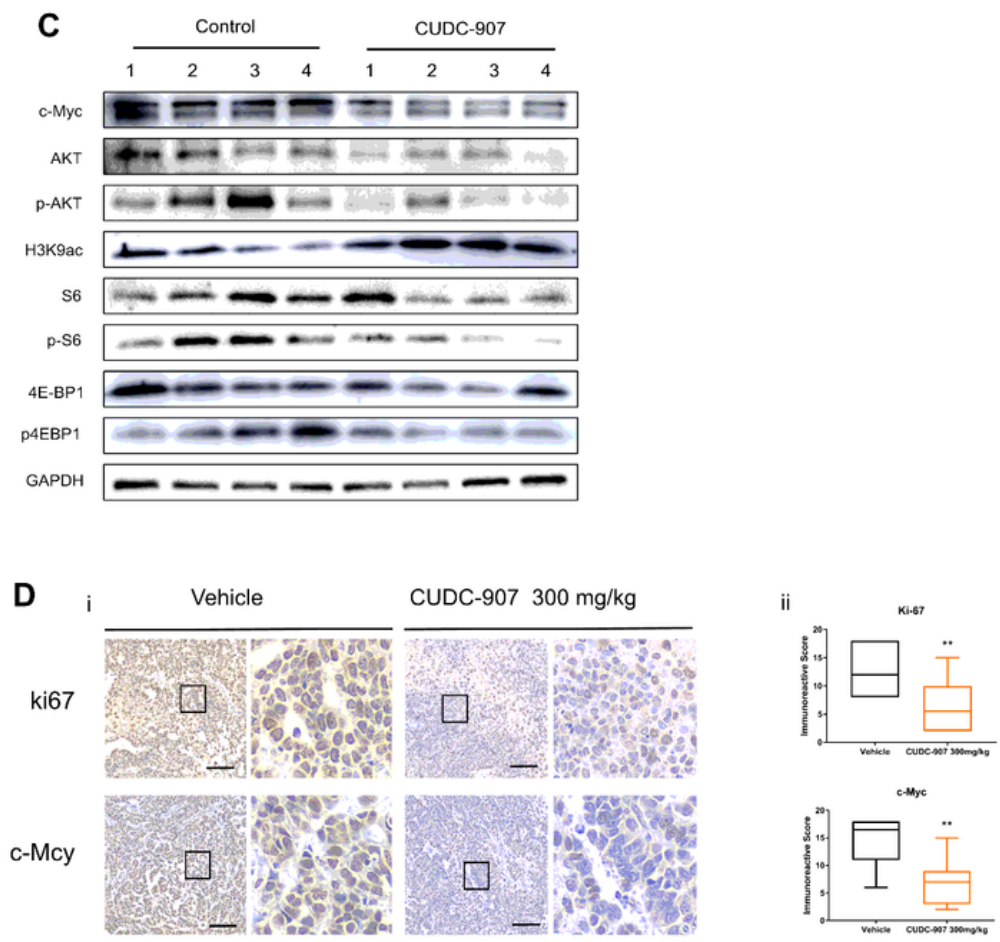

astape

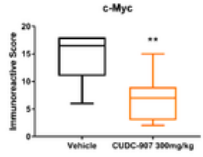

\section{Figure 6}

Western blotting of downstream regulated protein of CUDC-907. A The expression of c-Myc is regulated by HDAC and $\mathrm{PI3K} / \mathrm{AKT} / \mathrm{mTOR}$ pathway [18], and both can be inhibited by CUDC-907. B Expression level of proteins including c-Myc, pAKT, p-S6, p-4EBP1 and the acetylation of histone.in HCC cell lines affected by CUDC-907 were detected by western blotting. GAPDH was set as the internal reference. C. Expression level of proteins (same as the proteins mentioned in Fig 6B) from xenografted tumor with or without treatment of CUDC-907 were detected. D Immunohistochemistry staining. (i) Representative images of Ki67 and c-Myc staining of xenografts treated with CUDC-907 or vehicle. (ii) Quantification of immunohistochemistry staining was calculated by immunohistochemistry score method. Ten random areas from four individually stained tumors were captured. ${ }^{\star} \mathrm{p}<0.05,{ }^{\star *} \mathrm{p}<0.001$. 
A

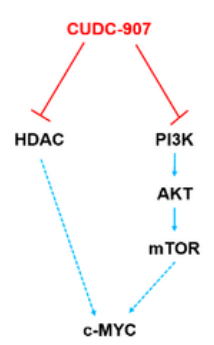

B

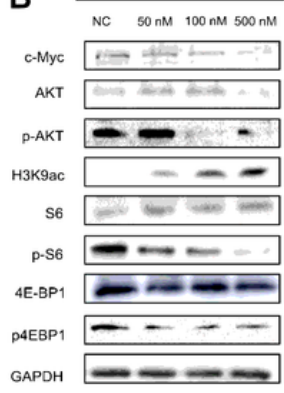

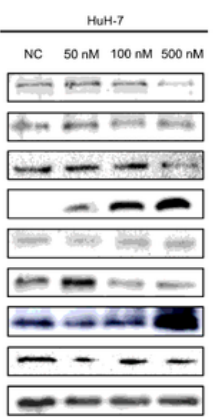
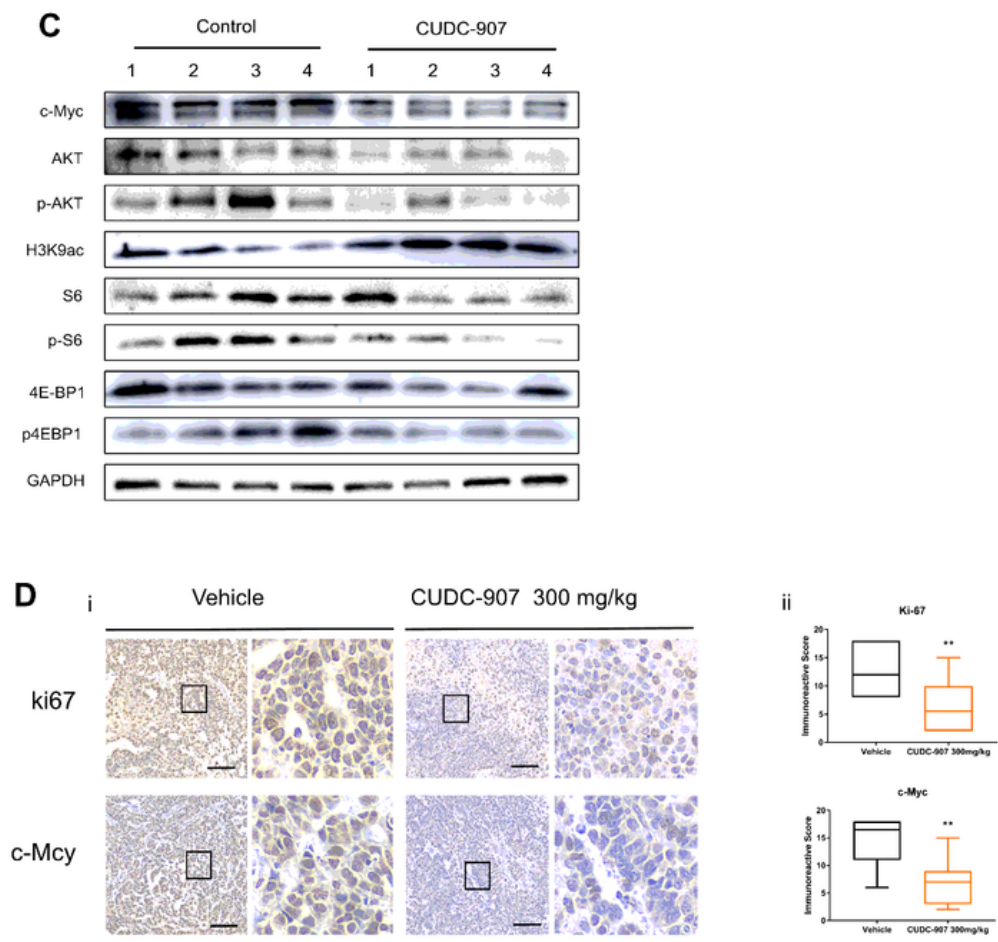

astape

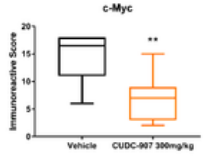

\section{Figure 6}

Western blotting of downstream regulated protein of CUDC-907. A The expression of c-Myc is regulated by HDAC and $\mathrm{PI3K} / \mathrm{AKT} / \mathrm{mTOR}$ pathway [18], and both can be inhibited by CUDC-907. B Expression level of proteins including c-Myc, pAKT, p-S6, p-4EBP1 and the acetylation of histone.in HCC cell lines affected by CUDC-907 were detected by western blotting. GAPDH was set as the internal reference. C. Expression level of proteins (same as the proteins mentioned in Fig 6B) from xenografted tumor with or without treatment of CUDC-907 were detected. D Immunohistochemistry staining. (i) Representative images of Ki67 and c-Myc staining of xenografts treated with CUDC-907 or vehicle. (ii) Quantification of immunohistochemistry staining was calculated by immunohistochemistry score method. Ten random areas from four individually stained tumors were captured. ${ }^{\star} \mathrm{p}<0.05,{ }^{\star *} \mathrm{p}<0.001$. 